\title{
PROCODER: A professional tape control, coding, and analysis system for behavioral research using videotape
}

\author{
JON TAPP and TEDRA WALDEN \\ Vanderbilt University, Nashville, Tennessee
}

\begin{abstract}
PROCODER is a software system for observing and coding events that have been recorded on videotape. The system uses a personal-computer-based tape controller to control a VHS tape while observations are recorded. Frequencies of events, durations of events, and calculations of interobserver agreement of events or intervals are included. Data can be output in ASCII format for use with other statistical programs. A sample study in which the system is used is described as well.
\end{abstract}

Events or interactions between actors in natural or experimental settings, as well as quantification of behavior types, are of interest in many aspects of behavioral research. With the development of increasingly sophisticated theory and methodology, many scientists turn at least occasionally to coding micro-units of behavior when detailed analyses of behavior of one or more persons are called for. In our work with infant social referencing (described briefly below), we have been interested in observing and/or rating behavior of three different types: (1) occurrences of a number of behaviors (as well as the time of each occurrence), (2) ratings of affect within short intervals, and (3) continuous changes as they occur in infants' visual attention.

Live coding is limited in the number of behaviors that can be observed and recorded simultaneously, therefore many of our observations are now recorded on videotape. Videotaped records allow us to view a single episode or session repeatedly, either in real time or slow motion, and thus record a greater number of events, record many simultaneous events, or code details of behavior with greater precision and specificity than would otherwise be possible. Furthermore, videotape allows us to consider time as a factor in much more detail than can be achieved without tape. Studies may require counting occurrences of target behaviors (event coding) or recording descriptors of behavior occurring within certain units of time (interval coding). PROCODER was designed at Vanderbilt University's Kennedy Center for Research on Education

\footnotetext{
This research was supported in part by Grants NICHD P30HD15052 and NSF BNS9109634. We gratefully acknowledge the contributions of Abigail Baxter, David Fiel, Carol Hamlett, Willie Horn, Linda Knieps, and Tamara Ogan to this project. Correspondence should be addressed to Jon Tapp, 306 Liberty Lane, Lavergne, TN 37086.

Note: The author has a direct financial interest in some of the software described in this paper.-Editor
}

and Human Development to facilitate acquisition of such data from videotaped sessions.

PROCODER uses industry-standard time coding and videotape editing technology to facilitate data acquisition by allowing the user to control and code a tape by making simple keystrokes at a computer console. Codes are checked for validity during the coding process to help eliminate coding errors. The program can code videotape events to $1 / 30$-sec (single frame) accuracy either by using onset and offset frame numbers (time) or by playing an interval of any designated length (number of frames) and recording codes for each interval. Analyses of the frequencies and durations of events and calculations of interobserver agreement of events or intervals are available. In addition, the program outputs coded data in an ASCII file format for more extensive analysis with other statistical programs.

\section{Hardware Requirements for PROCODER}

The program uses an IBM PC or compatible personal computer with at least $640 \mathrm{~K}$ of RAM. Although the program can run on an 8088/86-based machine with two floppy disks, an 80286- or 80386-based machine with a hard drive is recommended because of greater speed and faster return of time code numbers to the computer. The current version of the program uses a BCD 4000 or BCD 232c tape controller (available from BCD Associates, Inc., 7510 Broadway, Suite 205, Oklahoma City, OK 73116). A VHS tape player supported by BCD hardware and a monitor on which the coder observes the videotape are also required, as is a computer monitor, which displays codes as they are entered and reviews codes previously entered. The program can also be run without a tape control system, but it is not generally recommended because the user would be required to enter time values manually.

PROCODER has been tested and developed using Panasonic edit-quality tape decks. The program is being ex- 
panded for other edit decks and controllers: Contact the first author for a current list. Interested parties should also contact the controller manufacturer for a complete list of supported tape decks.

\section{Time Code Support}

Videotape edit controllers work by taking over the functions that are normally available on the front panel or remote control unit of the VCR (e.g., Play, Fast Forward, Rewind). To provide frame-accurate control, edit controllers write a frame number, called a time code, on each frame of the videotape. On VHS tape, the time code traditionally has been written on one of the two audio tracks. More recently, however, technological improvement has made it possible for the time code to be written on the video portion of the tape between the actual video frames.

The industry-standard format for writing time code, whether to the audio or the video portion of a tape, is commonly referred to as SMPTE time coding (Society of Motion Picture and Television Engineers). The method used in writing the time code specifically to the video portion is referred to as vertical interlaced time coding, or VITC. The VITC method has the advantage of noninterference with the audio tracks, greater long-term stability, and better frame accuracy. PROCODER can use (1) the SMPTE format for time coding on either of the two audio tracks, (2) the SMPTE format with the VITC method for time coding on the video portion of the tape, or (3) BCD's dedicated format for time coding on either the audio or the video portion of the tape.

\section{Steps in Using PROCODER}

1. Record the session on videotape.

2. Time code the tape. With PROCODER, a time code can be written, by means of an audio dub, onto the videotape in any of the above formats either after recording or as the original tape is being duplicated.

3. Develop the coding scheme. In PROCODER, a code file is defined that contains the codes that designate events in your study. Codes consist of a string of up to 8 characters, a 25-character description of the code, and a status variable used in on-line checking. There are three types of codes: (a) codes for events or behavior events (codes that have an onset and offset time), (b) codes used in designating intervals, and (c) codes that have a rating associated with them (used to rate or make a judgment about an event's qualities or characteristics). The status variable attached to each code, in conjunction with the segment file (discussed below), allows a coder to identify segments of each scene in which each code can legally occur. Thus, the tape is segmented according to some criteria important to the study (e.g., trial:1/ITI/trial $2 /$ ITI/trial $3 / \ldots$ ). This helps minimize coding errors. The codes are kept in a separate code file in the same directory with the program.

4. Segment the tape. Most experimental studies do not require an entire videotape to record a session; therefore, a given videotape may contain several sessions or parts of sessions that are of interest. The segment file, which is kept in a separate file from the event data, allows a coder to identify segments that narrow the part of the tape to be coded. Segmenting allows a coder to use tape more efficiently by putting many segments on one tape and minimizes time shuttling the videotape during coding, since frame numbers in each segment are relatively close.

Segment files consist of a starting frame number, up to three breakpoints, an ending frame, and a condition code for the segment, which is used for later quantification of any attribute the coder wants to rate. The breakpoints and condition codes allow designation of sets of allowable codes for the different portions of a segment. For example, a code with a status variable of zero is allowed at any point in a segment, whereas a status code of one is allowed only between the beginning of the segment and breakpoint 1 , and so on.

Coders mark tape segments while viewing the tape by depressing a "mark" key on the computer keyboard. The mark key puts the current frame number read from the tape into the currently selected time area on the screen. For example, to mark the beginning of a segment, the cursor is moved to the start-frame position on the screen for the current segment and the mark key is pressed when the tape reaches the beginning of the segment. The tape is controlled from a pop-up menu of tape controls or by using one of the many defined "hot" keys (e.g., "Alt-P" for play). Hot keys are single keystroke commands to control the tape player and read in frame numbers without looking away from the tape.

5. Code or rate the events. After the tape has been time coded and segmented, a coder enters codes for the events of interest. As mentioned above, for interval coding, a preselected number of frames are played from the beginning of a segment, the player stops and waits for a code entry, which is checked against the allowable codes designated for that segment, and then the next interval is played, and so on. If event codes are used, the event onset and offset frame numbers are marked as the tape is playing (in real time or slow motion). Event codes can contain a rating value in either event or interval coding modes.

Event codes are entered in a way similar to the designation of segment marks by locating the cursor at the event onset and pressing the mark key on the keyboard. The tape can be controlled either by using the tape control menu or with hot keys. An event can be played and replayed for verification by making a single keystroke. Events can be entered or changed independently of the time code entries. A single frame advance command is included for those who need to code data frame by frame. The tape can be either stopped or in any state of motion while events are entered or changed. Usually, the event times are marked, and the descriptor codes are entered later. If a code is entered that is not contained in the code file or is not valid during the current time segment (as defined in the code file) a beep sounds, an error message is displayed, and the code is not accepted for entry.

6. Analyze the data. After coding the tape, PROCODER can compute the frequency and duration of all events or 
intervals coded. PROCODER checks the reliability of observations by calculating interobserver agreement between separately coded data located in two separate data files, using the method described in MacLean, Tapp, and Johnson (1985). Interobserver agreement on both frequency and duration is provided by the program. Frequency of interobserver agreement on coding events is computed by using a user-selected time window (in frames) around one coder's events and scoring agreements with another coder's events within the same time interval. All codes that are not matched as agreements are counted as disagreements. To assess interobserver agreements on duration, interval, and rating data, both Cohen's Kappa (Cohen, 1960) and an agreement ratio are computed for each code by examining the state of each code at each frame that is defined in the segment file.

A merged output file in standard ASCII format can be produced for use in any statistical analysis program. The file can either be in a fixed-space format or in fields separated by commas. The program can put the segment data, the event data, and/or the interval data into the same merged output file. Each record of the file contains an identifying string (optional), the filename that contained the data (optional), the condition code for the segment (optional), the event code string, the starting frame number for the event, and the ending frame number. The records in the file are sorted by starting frame number before the output file is written. The output file is opened for appending so that multiple event files can be added to the end of an existing output file.

\section{The User Interface}

PROCODER is menu-driven and intuitive in its design. The menu choices are descriptive and can be selected by using the arrow keys and return key or by selecting the highlighted key of choice. Context-sensitive help is obtained at any point by pressing the Fl key. The program should be extremely easy to install and to use if the stepby-step instructions provided with the program are followed. Persons with a working knowledge of VHS tape decks and a basic understanding of MS-DOS-based personal computers should be able to use the program without any assistance.

\section{Unique Features of the Program}

Although PROCODER is similar to tape-editing and list-management programs, it differs from these programs in fundamental ways. Tape-editing programs are used to store previously recorded scenes for use in assembling a finished edited tape from a tape with scenes in random order. An edit program uses and controls two tape decks to make a finished copy. List-management systems perform an archival role-that is, they store lists of scenes from the videotape for storage and retrieval. For example, a museum may store tapes of artwork in order to identify works at a later date, or a plastic surgeon may store tapes of prior surgery patents to show his or her work to new patients.
PROCODER is similar to edit programs because the user can mark segments of tape as events with a starting frame and an ending frame number (commonly called an edit list) and can enter a description of each event (the codes in PROCODER), but this is where the similarity ends. The program is one of the first developed specifically for coding behavioral events. The ideas used in PROCODER have been developed out of years of experience by investigators at the Kennedy Center. Each entry is checked when it is entered with the on-line checking to verify that it is a permissible entry at that point, preventing coding errors caused by mistaken keystrokes. Different codes can be designated as permissible during different segments of the tape. If an illegal code is entered, an alert beep sounds, an explanation is displayed as to why the code is illegal, and the code is not entered into the data stream. A new code must be entered at that point. The creation of segment files, definition of codes with ratings, the ability to use both event codes and interval codes, and the built-in analyses of reliability make PROCODER extremely useful for coding behavioral data.

\section{A Sample Study Using PROCODER}

A variety of research questions could make use of the kinds of data described above. PROCODER was developed and first used to obtain data on infant social referencing. In social referencing, one person seeks information about how to interpret or react to an event from another person whose facial expressions, voice characteristics, body movements and gestures, and verbal content may convey messages of danger, threat, approach, disgust, surprise, and so on.

We have come to appreciate that infants as young as 1 year of age engage in social referencing and use information from caregivers' reactions to events to regulate their own behavior and affect. We have been studying the development of social referencing from early infancy through preschool in normally developing and mentally retarded children (Walden \& Baxter, 1989; Walden, Knieps, \& Baxter, 1991; Walden \& Ogan, 1988). In a series of experiments, parents were trained to give various emotional signals corresponding to expressions such as happiness or fear in order to observe how (and whether) the child's behavior and affect were influenced by the parent's message.

We required detailed coding of several aspects of emotionality of parents' messages, details of infants' behavior (e.g. , approach and avoidance of the stimulus or smiling and crying), and ratings of infants' affect within 10-sec intervals that continued throughout the session. The design of the study included several different types of trials during a session, and repeated trials within those trials (trials within trial blocks). Many passes through the data were required to code the behavior of both interaction partners on the many different behaviors (about $20 \mathrm{~h}$ of coding was required for each 20 -min session).

Event coding was used to record occurrences of specific behaviors. Interval coding was used to code infant 
affect. A continuous record of changes in infant states of visual attention across the session was obtained by using the event-coding facility-that is, the onset of each change in state of attention was recorded as an event, with the onset of a new state serving as the offset of the previous state of attention. Different codes were allowable during different portions of the sessions and for coding events or intervals. Our on-line checking of allowable codes was sensitive to changes in this parameter, even when coders were not, and prevented these mistakes from entering the data and subsequently having to be discarded (a critical problem in sequential-type analyses).

Data obtained on each pass were linked via PROCODER with data from other passes and organized to occur in chronological order by onset time. This eliminated a time-consuming task that is often done by hand. Many coders worked on various coding tasks both within each subject and across the many subjects in these studies, and the linking of all the separate codings was required.

By using data coded with PROCODER, we have discovered a great deal about the development of social referencing from infancy through childhood. We have been able to observe changes in infants' regulation of their behavior and affect and in the use of emotional information from parents as infants mature. Changes in parents' communicative messages also occur as children get older and more verbal. We have found also that parents and their infants with mental retardation differ from normally developing dyads in their patterns of interaction in social referencing situations, because children with retardation do not regulate their behavior and affect based on parents' messages, as do their normally developing peers.

In many cases, subtle but significant behaviors have indicated the answers to research questions that we have asked. In addition, links between parent behavior and child behavior often explain more than behaviors of either member of the dyad alone (Baxter \& Walden, 1992). With PROCODER, sequences of behavior of one or more interaction partners are preserved, thus making sequential anal- ysis possible as well. Without the hardware and software technology that allowed us to use sophisticated behavior coding techniques, addressing this kind of research question would not have been possible.

\section{Availability}

The PROCODER package includes the software disk and manual only; the computer and videotape hardware must be purchased separately. The program costs $\$ 450$, which includes up to an hour of support from the first author during nonbusiness hours. The software and manual are not available through e-mail. The first author is licensed by an agreement with Vanderbilt University to distribute the program to the general public. Correspondence should be addressed to Jon Tapp and Associates and sent to Jon Tapp, 306 Liberty Lane, Lavergne, TN 37086 (Bitnet: tappjt@vuctrvax; Internet: tappjt@ ctrvax.vanderbilt.edu).

\section{REFERENCES}

BAXTER, A., \& WALDEN, T. (1992). Social referencing as a function of infants' initial affective reactions. Manuscript submitted for publication.

CoHEN, J. (1960). A coefficient of agreement for nominal scales. Educational \& Psychological Measurement, 20, 37-46.

Maclean, W. E., JR., TAPP, J. T., Johnson, W. L. (1985). Alternate methods and software for calculating interobserver agreement for continuous observation data. Journal of Psychopathology \& Behavioral Assessment, 7, 65-73.

WALDEN, T., \& BAXTER, A. (1989). The effects of age and familiarity of setting on infant social referencing. Child Development, 60, 1511-1518.

WAlden, T., KNIEPS, L. J., \& BAxter, A. (1991). Contingent provision of social referential information by parents of children with and without developmental delays. American Journal on Mental Retardation, 96, 177-187.

WaLDEN, T., \& OGan, T. (1988). The development of social referencing. Child Development, 59, 1230-1240.

(Manuscript received February 10, 1992; revision accepted for publication August 10, 1992.) 